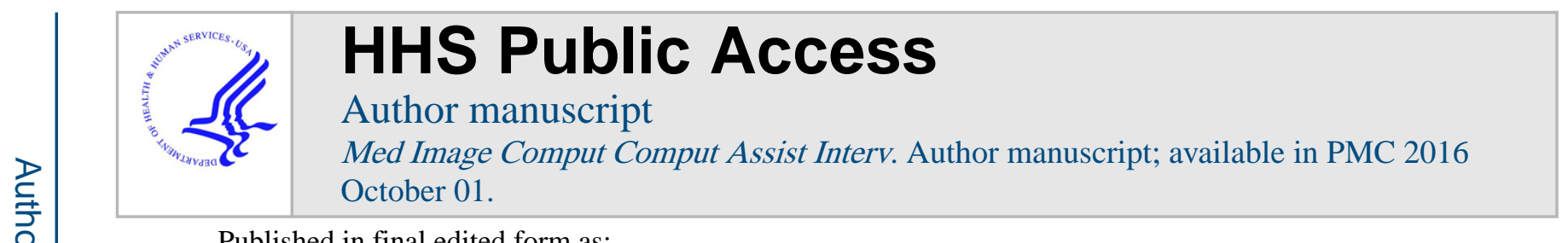

Published in final edited form as:

Med Image Comput Comput Assist Interv. 2015 October ; 9350: 152-159.

\title{
Automatic graph-based localization of cochlear implant electrodes in CT
}

\author{
Jack H. Noble and Benoit M. Dawant \\ Dept. of Elect. Eng. and Comp. Sci., Vanderbilt University, Nashville, TN USA
}

\begin{abstract}
Cochlear Implants (CIs) restore hearing using an electrode array that is surgically implanted into the cochlea. Research has indicated there is a link between electrode location within the cochlea and hearing outcomes, however, comprehensive analysis of this phenomenon has not been possible because techniques proposed for locating electrodes only work for specific implant models or are too labor intensive to be applied on large datasets. We present a general and automatic graph-based method for localizing electrode arrays in CTs that is effective for various implant models. It relies on a novel algorithm for finding an optimal path of fixed length in a graph and achieves maximum localization errors that are sub-voxel. These results indicate that our methods could be used on a large scale to study the link between electrode placement and outcome across electrode array types, which could lead to advances that improve hearing outcomes for CI users.
\end{abstract}

\section{Keywords}

cochlear implant; optimal path; graph; segmentation

\section{Introduction}

Cochlear implants (CIs) are considered the standard of care treatment for profound hearing loss [1]. CIs use an array of electrodes surgically implanted into the cochlea to directly stimulate the auditory nerve, inducing the sensation of hearing. Although CIs have been remarkably successful, speech recognition ability remains highly variable across CI recipients. Research has indicated there is a strong link between electrode location within the cochlea and hearing outcomes [2-6]. However, comprehensive analysis of this phenomenon has not been possible because precise electrode position has been unknown. Electrode position is generally unknown in surgery because the array is blindly threaded into a small opening of the cochlea. To analyze the relationship between electrode position and outcome, several groups have proposed post-operative imaging techniques. This has included imprecise measures such as ones that can be visually assessed in CT images, e.g., is the array entirely within one internal cavity of the cochlea, the depth of insertion of the last electrode, etc. [2-6]. These studies have concluded that electrode position and outcomes are correlated but are conflicting or inconclusive regarding what specific factors are important because dataset sizes were limited and electrode positions were not precisely determined. Da-taset size has been limited due in part to the amount of manual effort that must be undertaken to analyze the images. Another application where precise electrode localization is important is for systems being developed that use electrode position to select better CI 
processor settings [7]. Such systems have been shown to significantly improve hearing outcomes compared to standard clinical results. In the current work, we propose a fully automatic approach for localizing CI electrodes in CT images. Such an approach could permit localizing electrodes on large numbers of datasets to better analyze the relationship between electrode position and outcome, which may lead to advances in implant design or surgical techniques. It could also automate the electrode localization process in systems designed to determine patient-customized CI settings, such as the one proposed in [7], reducing the technical expertise required to use such technologies and facilitating transition to large scale clinical use.

Figure 1 shows an example of an electrode array in CT. Localizing the electrodes in CT images is difficult because, as seen in figure $1 \mathrm{~b}$, finding a threshold to isolate the electrodes is not possible due to the presence of the connecting wires and because adjacent bony tissue voxels are assigned erroneously high intensity in image reconstruction due to beam hardening artifacts caused by the metallic electrodes. Another issue is that there is often no contrast between the electrodes, even when the CTs are acquired at very fine slice thickness and resolution. Where there is no contrast, the challenge is to identify the center of each of the contacts, and where the electrodes are separated, the challenge is to determine how the different groups of contacts are connected. Previous approaches [8,9] require electrodes to be grouped to determine their connectivity and thus are not applicable to many electrode types that have wider contact spacing. Our solution is to simultaneously identify the centers of the electrodes and their connectivity using a novel, graph-based path finding technique. Our approach can handle varying degrees of contrast between electrodes and thus is applicable to many electrode types. Our results, presented and discussed in Sections 3 and 4, will show that this fully automatic approach can reliably be applied to clinical images.

\section{Methods}

The automatic segmentation method we propose is outlined in Figure 1. As can be seen in the figure, the first step (1) involves coarsely estimating the location of the region of interest (ROI), which is a local region $\sim 30 \mathrm{~cm}^{3}$ around the cochlea. This is done through registration with a known volume. The subsequent processing steps are then performed solely within the ROI. The next step (2) is to identify a set of points in the ROI representing candidate electrode locations. This is required for the following coarse electrode localization step (3) in which a graph search technique is used to identify points corresponding to electrodes from among those candidates using an intensity and shape-based cost function. Once the electrodes are coarsely localized, the next step (4) is to refine their positions using a subsequent path finding step. The following subsections detail this approach.

\subsection{Data}

The images in our dataset include images from 20 subjects acquired with a Xoran $\mathrm{xCAT}^{\circledR}$. The images have voxel size $0.4 \times 0.4 \times 0.4 \mathrm{~mm}^{3}$. Half of the subjects were implanted with Advanced Bionics Mid Scala arrays and the other half with Advanced Bionics 1J arrays. Since the Mid-Scala arrays have little contrast between electrodes in CT due to close electrode spacing $(<1 \mathrm{~mm})$ whereas the $1 \mathrm{~J}$ arrays typically have strong contrast between 
electrodes in CT due to larger electrode spacing $(>1 \mathrm{~mm})$, these two array types are reasonably representative of the types of image contrast variations seen among the many FDA approved array models. Both Mid-Scala and 1J arrays are composed of 16 active contacts and 1 non-stimulating marker contact. The dataset of 20 subjects was subsequently divided into a training and testing set of 10 subjects each. The training and testing sets had equal number of each electrode type. The training set was used for parameter tuning and the algorithm was validated on the testing set with fixed parameters. A ground truth electrode localization solution for each dataset was created by manual localization of the contact positions by an expert. For the testing set, average contact positions from three manual localization sessions were used for ground truth.

\subsection{Candidate Selection}

After identifying the ROI, the first step in our approach is to select points that represent candidate electrode locations. The following process will select from among these candidates the points that coarsely correspond to the electrodes. To select the candidates, we first create a binary mask by thresholding the image at the intensity corresponding to the top $\Psi_{1}=0.08 \%$ of the cumulative histogram of the ROI image. This threshold represents a value that is low enough to ensure no contacts are discared from the result, which is vital for the success of the following procedures, but not so low that an excessively large volume of noncontact voxels are included in the result, which is also important to prevent the following steps from becoming too complex. An example result of this process is shown in transparent gray in Figure 1b. After thresholding, the set of connected components, each of which corresponds to one or multiple electrodes or background tissue, is voxel thinned by extracting its medial axis [10] to further prune the results. The set of candidate points are chosen as the voxels $\{\boldsymbol{v}\}_{1}^{V}$ that compose this set of $M$ medial axes $\{\boldsymbol{m}\}_{1}^{M}$. This procedure typically results in $V=\sim 80$ candidate points. Example results of this process where medial axes were extracted from $M=4$ connected components are shown in red in Figure $1 \mathrm{~b}$.

\subsection{Coarse Electrode Localization}

After the candidates are selected, a graph-based procedure is used to localize the electrodes. Each candidate point is treated as a node in a graph, and the path finding algorithm aims to find the sequence of $\boldsymbol{L}=17$ nodes, where $\boldsymbol{L}$ is the number of electrodes, that obeys node neighbor constraints and minimizes an intensity and shape-based cost function. The path finding algorithm pseudocode is shown in Algorithm 1. The algorithm begins with a seed node, chosen as discussed below, that represents the position of the first electrode. Then, the $\boldsymbol{L}-1$ remaining nodes are determined by growing a tree of candidate paths $\{\boldsymbol{p}\}$ that stem from the seed node. At each ith iteration in the main for-loop, in the Grow stage, the set of new candidate paths $\{\boldsymbol{q}\}$ of length $i+1$ are found as the combination of all possible one-node extensions of the set of length $i$ candidate paths $\{\boldsymbol{p}\}$ to their permissible child nodes $\{\boldsymbol{c}\}$ This potentially large set of candidate paths $\{\boldsymbol{q}\}$ is then reduced in the Prune stage to keep for the next iteration only the $\boldsymbol{P}$ candidate paths that correspond to the lowest cost. The output of the algorithm is the lowest cost length $\boldsymbol{L}$ path, $\left\{\boldsymbol{p}_{\boldsymbol{1}}\right\}$. While our technique provides no guarantee of finding a global optimum, the advantage over techniques like Dijkstra's algorithm [11] is that, as shown below, it permits non-local and shape-based constraints. Shape constraint is 
needed because a path composed of electrodes will not generally optimize an intensity-only cost function due to electrode image intensity inhomogeneity.

The child nodes $\{\boldsymbol{c}\}$ of a path $\boldsymbol{p}$ of length $i$ are chosen to be the nodes that obey reasonable constraints. First, they must obey the hard constraint

$$
\frac{1}{\alpha_{2}} d_{i}<\left\|\boldsymbol{p}_{i}-\boldsymbol{c}\right\|_{2}<\alpha_{2} d_{i}
$$

where $d_{i}$ is the a priori expected distance between electrodes $i$ and $i+1$, which is specific to the model of the array; and $a_{2}=2.0$. This constraint only permits child nodes with distance to the candidate path's endnode $\boldsymbol{p}_{\mathrm{i}}$ that falls within bounds of the expected distance between electrodes. The child nodes must obey further hard constraints that that $\boldsymbol{c} \notin \boldsymbol{p}$, i.e, $\boldsymbol{c}$ is not already in the path; $\boldsymbol{c} \notin\{\boldsymbol{m}\} \forall$ medial axes $\boldsymbol{m}$ that do not include $\boldsymbol{p}_{\mathrm{i}}$ but do include any of the points in $\{\boldsymbol{p}\}_{1}^{i-1}$, which is a constraint that prevents the path from containing disjoint groups of nodes that belong to the same medial axis $\boldsymbol{m}$ (defined above in Section 2.2), i.e., a medial axis cannot be revisited by a path; and a final constraint that if $\boldsymbol{c}, \boldsymbol{p}_{\mathrm{i}}, \boldsymbol{p}_{\mathrm{i}-1} \in \boldsymbol{m}$, then $\boldsymbol{c}$ is a valid child node only if $\boldsymbol{c}, \boldsymbol{p}_{\mathrm{i}}$, and $\boldsymbol{p}_{\mathrm{i}-1}$ are monotonically ordered in medial axis $\boldsymbol{m}$, which is a constraint that prohibits paths that are following a given medial axis from changing directions on that curve. The underlying assumption forming the basis for last two medial axis-based constraints is that the ordering of electrodes belonging to the same medial axis should match their order in the final path.

The cost function for a child node that obeys the above hard constraints is defined as a combination of intensity and shape-based cost terms,

$$
\operatorname{Cost}_{1}(\boldsymbol{c}, \boldsymbol{p})=\mathrm{C}_{\mathrm{I}}(\boldsymbol{c}, \boldsymbol{p})+C_{S}(\boldsymbol{c}, \boldsymbol{p}),
$$

where

$$
\begin{gathered}
\mathrm{C}_{\mathrm{I}}(\boldsymbol{c}, \boldsymbol{p})=\frac{\left(I_{\max }-I(c)\right)}{2000} \times\left\{\begin{array}{cc}
\alpha_{3} & i \geq \alpha_{4} \\
1 & \text { else }
\end{array}, \quad, 3\right) \\
\mathrm{C}_{\mathrm{S}}(\boldsymbol{c}, \boldsymbol{p})=\alpha_{5}\left(1-\left\{\begin{array}{cc}
\operatorname{Cos}(\boldsymbol{c}, \boldsymbol{p}) & \operatorname{Cos}(\boldsymbol{c}, \boldsymbol{p})<0.5 \\
1 & \text { else }
\end{array}\right)+\left\{\begin{array}{cc}
-\alpha_{6} \operatorname{Dst}(\boldsymbol{c}, \boldsymbol{p}) & \operatorname{Dst}(\boldsymbol{c}, \boldsymbol{p})<0 \\
\alpha_{7} \operatorname{Dst}(\boldsymbol{c}, \boldsymbol{p}) & \text { else }
\end{array},\right.\right. \\
\operatorname{Cos}(\boldsymbol{c}, \boldsymbol{p})=\frac{\left(\boldsymbol{c}, \boldsymbol{p}_{i}\right) \cdot\left(\boldsymbol{p}_{i}-\boldsymbol{p}_{i-1}\right)}{\left\|\boldsymbol{c}-\boldsymbol{p}_{i}\right\|\left\|\boldsymbol{p}_{i}-\boldsymbol{p}_{i-1}\right\|}, \operatorname{Dst}(\boldsymbol{c}, \boldsymbol{p})=\left\|\boldsymbol{c}-\boldsymbol{p}_{i}\right\|_{2}-d_{i},
\end{gathered}
$$

$I_{\max }$ is the maximum intensity of the ROI image, $I(\boldsymbol{c})$ is the intensity of the image at $c, i$ is the length of path $\boldsymbol{p}, d_{i}$ is defined as in Eqn. (1), and $a_{3-7}$ are set to 0.1, 14, 1.0, 5.2, and 2.0 after training. Eqn. (3) assigns lower cost to child nodes that correspond to higher image intensity. The conditional term decreases the importance of image intensity for the last $\boldsymbol{L}+1$ $-a_{4}$ electrodes in the path. This term is included because the last few electrodes are typically less bright in CT because fewer internal wires extend to the end electrodes 
compared to those at the base. The first term in the shape cost function, Eqn. (4), is a smoothness term that punishes child nodes that would create a bend sharper than $45^{\circ}$ if added to $\boldsymbol{p}$. The second term punishes child nodes that are not the expected distance from $\boldsymbol{p}_{i}$.

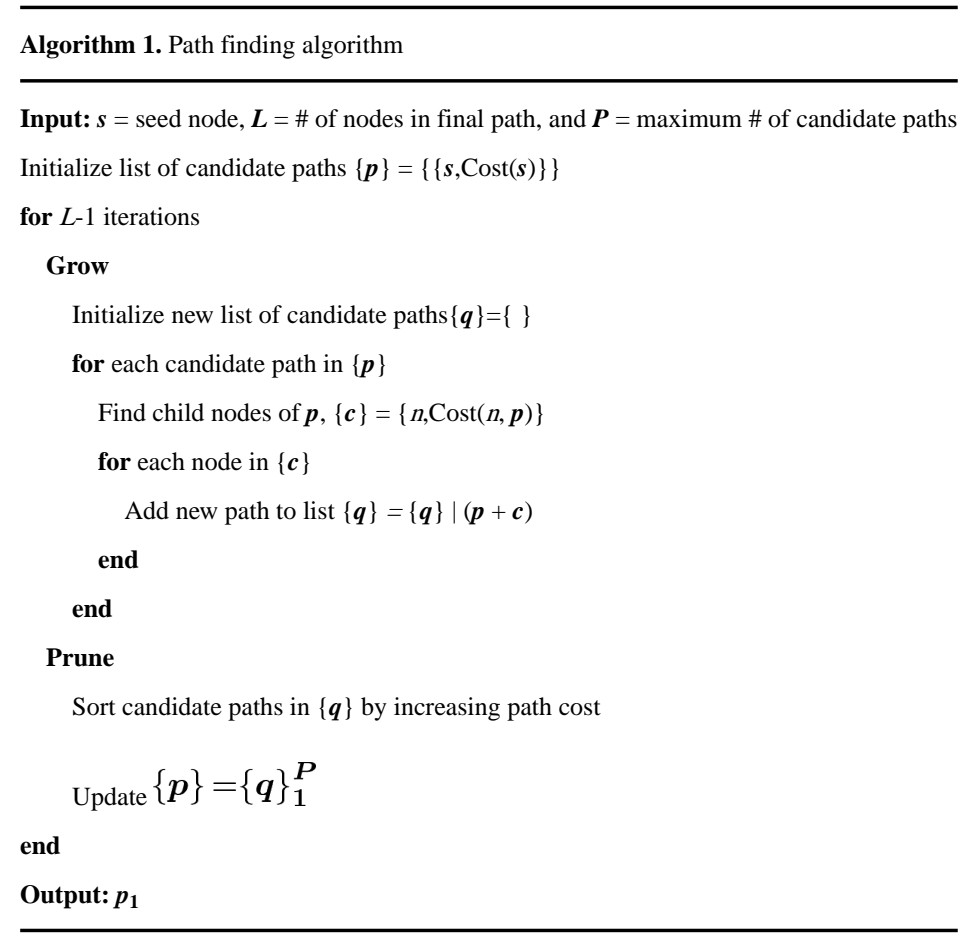

With the cost function and neighbor constraints defined, Algorithm 1 is executed (with $\boldsymbol{P}=$ $500)$ to locate the coarse position of each electrode. Each of the $\boldsymbol{V}$ candidate points in $\{\boldsymbol{v}\}$ (see Section 2.2) could potentially correspond to electrode 1 . Thus, we treat each point in $\{\boldsymbol{v}\}$ as a seed node and execute Algorithm $1 \boldsymbol{V}$ times in parallel, once for each of those seeds. Since the algorithm is pose invariant, there is potential to find low cost paths that are ordered undesirably from end to base. Such paths are detected using the orientation of the cochlea, which is known from the registration with the know volume, and these are discarded from the resulting set of $\boldsymbol{V}$ paths. The final path is then selected from among the remaining paths as the one with minimum cost.

\subsection{Electrode Localization Refinement}

The above process is able to only coarsely identify electrode location due to imperfect selection of candidate points $\{\boldsymbol{v}\}$ and the fact that the above process does not permit subvoxel precision. Thus, we use a secondary path finding procedure to refine the resulting coarse path. In this step, a set of nodes are defined by sampling a fine rectangular grid of points around each of the $\boldsymbol{L}$ coarse electrode estimates, $\left\{\boldsymbol{l}_{i}\right\}_{i=1}^{\boldsymbol{L}}$, as $\{\boldsymbol{n}\}^{i}=\left\{\boldsymbol{l}_{i}+\alpha_{8}[x, y, z]\right\}_{x, y, z \in\left[-\alpha_{9}, \alpha_{9}\right]}$, where $\alpha_{8}=0.12 \mathrm{~mm}$ and $\alpha_{9}=3$. The path finding step aims to refine the estimated position $l_{i}$ of each $i$ th electrode with one of the nearby candidates $\{\boldsymbol{n}\}^{i}$. The cost function is defined as 


$$
\operatorname{Cost}_{2}(\boldsymbol{c}, \boldsymbol{p})=-G_{\sigma}(I(\boldsymbol{c}))+\left\{\begin{array}{cc}
-\alpha_{10} \operatorname{Dst}(\boldsymbol{c}, \boldsymbol{p}) & \text { Dst }(\boldsymbol{c}, \boldsymbol{p})<0 s \\
\alpha_{11} \operatorname{Dst}(\boldsymbol{c}, \boldsymbol{p}) & \text { else }
\end{array}\right\}
$$

where $G_{\sigma}(I(\boldsymbol{c}))$ is the Gaussian filter response of the image at $\boldsymbol{c}$ with $\sigma=0.3 \mathrm{~mm}$, Dst $(\boldsymbol{c}, \boldsymbol{p})$ is defined as in Eqn. (5), $a_{10}=50$, and $a_{11}=20$. The first term in Eqn. (6) represents a scaled blob finding filter, and the second term applies the same geometric constraints on the spacing of the electrodes used in Section 2.3. The children nodes $\{\boldsymbol{c}\}$ of each node in $\{\boldsymbol{n}\}^{i}$ are simply defined as the set of all nodes in $\{\boldsymbol{n}\}^{i+1}$. All nodes in $\{\boldsymbol{n}\}^{1}$ are treated as seed nodes and Algorithm 1 is executed with $\boldsymbol{P}=500$. The resulting path refines the coarse electrode localization determined above and represents the automatically selected final position of the electrodes.

\subsection{Parameter Selection and Validation}

Initial values for all parameters were determined heuristically. Then, the parameters were optimized sequentially and iteratively until a solution was found where each parameter is locally optimal with respect to mean electrode localization error in the training dataset. The range around each value was updated when the selected value was changed. Step size was uniform over the range as shown in Fig2bc. The coarse localization step parameters were optimized first, followed by the refinement step parameters. After all parameters were fixed, the method was applied to the testing set and mean and max electrode localization errors were measured to validate the results.

\section{Results}

Figure 2 shows the results of the parameter tuning procedure. As can been seen in the figure, a local optimum of each parameter was successfully obtained on the training dataset. Several of the parameters in panel (b), $a_{2}, a_{3}, a_{5}$, and $P$, and several parameters in (c), $a_{10}, a_{11}$, and $P$, were relatively insensitive around the minimum and their tuning curves have regions that are nearly flat and have high overlap. Selection of the other parameters was more crucial to obtaining the most accurate results.

Table 1 shows the overall segmentation error results. As shown, on our testing set our method is able to achieve mean electrode localization errors of $0.13 \mathrm{~mm}$ for Mid-Scala arrays and $0.09 \mathrm{~mm}$ for $1 \mathrm{~J}$ arrays. These errors are slightly larger than intra-rater variability levels of 0.09 and $0.08 \mathrm{~mm}$ for Mid-Scala and $1 \mathrm{~J}$ arrays. The maximum error in locating any electrode over all ten testing datasets was $0.33 \mathrm{~mm}$. Given that the voxel size of these images is $0.4 \times 0.4 \times 0.4 \mathrm{~mm}^{3}$, our worst electrode localization error was sub-voxel. An example Mid-Scala result is shown in Figure 1c.

\section{Conclusions}

In this work, we have proposed a novel and fully automatic cochlear implant electrode localization method. Our experiments show that our method is extremely accurate, even when applied to clinical images. Compared to previous electrode localization techniques that are only applicable to certain electrode models $[8,9]$, the method we propose handles 
varying electrode array geometries and is generally applicable. Our approach is also fast, requiring only $3-5$ seconds of processing time after a $\sim 2$ minute registration procedure is used to localize the ROI. The novel path finding algorithm we propose permits shape constraint and could be used for other applications.

Future studies will involve testing our method with images acquired with different scanners and of subjects with different implant models. We also plan to apply our method to large numbers of datasets to facilitate studying how the location of individual electrodes correlates with outcomes with the goal of developing technologies that can improve hearing outcomes with CIs.

\section{Acknowledgments}

This research has been supported by NIH grants R01DC014037 and R21DC012620 from the NIDCD. The content is solely the responsibility of the authors and does not necessarily represent the official views of this institute.

\section{References}

1. Cochlear Implants. 2011National Institute on Deafness and Other Communication Disorders, No. $11-4798$

2. Verbist BM, Frijns JHM, Geleijns J, van Buchem MA. Multisection CT as a Valuable Tool in the Postoperative Assessment of Cochlear Implant Patients. Am J Neuroradiol. 2005; 26:424-429. [PubMed: 15709150]

3. Aschendorff A, Kubalek R, Turowski B, Zanella F, Hochmuth A, Schumacher M, Klenzner T, Laszig R. Quality control after cochlear implant surgery by means of rotational tomography. Otol Neurotol. 2005; 26:34-37. [PubMed: 15699717]

4. Skinner MW, Holden TA, Whiting BR, Voie AH, Brundsen B, Neely GJ, Saxon EA, Hullar TE, Finley CC. In vivo estimates of the position of advanced bionics electrode arrays in the human cochlea. Annals of Otology, Rhinology and Laryngology Supplement. 2007; 197:2-24.

5. Wanna GB, Noble JH, McRacken TR, Dawant BM, Dietrich MS, Watkins LD, Schuman TA, Labadie RF. Assessment of electrode placement and audiologic outcomes in bilateral cochlear implantation. Otol Neurotol. 2011; 32:428-432. [PubMed: 21283037]

6. Wanna GB, Noble JH, Carlson ML, Gifford RH, Dietrich MS, Haynes DS, Dawant BM, Labadie RF. Impact of electrode design and surgical approach on scalar location and cochlear implant outcomes. Laryngoscope. 2014; 124(S6):S1-7. [PubMed: 24764083]

7. Noble JH, Labadie RF, Gifford RH, Dawant BM. Image-guidance enables new methods for customizing cochlear implant stimulation strategies. IEEE Trans on Neural Systems and Rehabilitiation Engineering. 2013; 21(5):820-829.

8. Noble, JH.; Schuman, TA.; Wright, CG.; Labadie, RF.; Dawant, BM. Automatic Identification of Cochlear Implant Electrode Arrays for Post-Operative Assessment. In: Dawant, BM.; Haynor, D., editors. Medical Imaging 2011: Image Processing Proc of the SPIE conf on Med Imag. Vol. 7962. 2011. p. 796217

9. Zhao, Y.; Dawant, BM.; Labadie, RF.; Noble, JH. MICCAI 2014, LNCS. Vol. 8673. Springer; 2014. Automatic Localization of Cochlear Implant Electrodes in CT; p. 331-8.

10. Bouix S, Siddiqi K, Tannenbaum A. Flux driven automatic centerline extraction. Medical Image Analysis. 2005; 9:209-221. [PubMed: 15854842]

11. Dijkstra EW. A note on two problems in connexion with graphs. Numerische Mathematik. 1959; $1: 269-271$. 


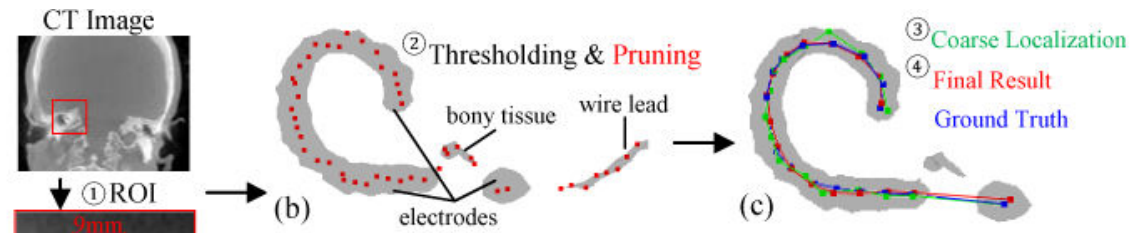

(a)

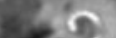

Fig. 1. Process flow chart. The ROI is shown in (a). In (b), example thresholding and pruning results are shown. In (c), coarse and refined electrode localization results are shown with the ground truth.

Fig. 1.

Process flow chart. The ROI is shown in (a). In (b), example thresholding and pruning results are shown. In (c), coarse and refined electrode localization results are shown with the ground truth. 


\begin{tabular}{cccc}
\hline \multicolumn{3}{l}{ Parameters Initial value Final value } & Range \\
\hline$\square \alpha_{1}$ & 0.08 & 0.08 & {$[0.03,0.13]$} \\
$\square \alpha_{2}$ & 2 & 2 & {$[0.5,3.5]$} \\
$\square \alpha_{3}$ & 0.1 & 0.1 & {$[0.05,0.55]$} \\
$\square \alpha_{4}$ & 14 & 14 & {$[7,17]$} \\
$\square \alpha_{5}$ & 1 & 1 & {$[0.5,1.5]$} \\
$\square \alpha_{6}$ & 4 & 5.2 & {$[3.2,7.2]$} \\
$\square \alpha_{7}$ & 2 & 2 & {$[0.5,3.5]$} \\
$\square P$ & 500 & 500 & {$[100,900]$} \\
\hline$\square \alpha_{8}$ & 0.12 & 0.12 & {$[0.02,0.22]$} \\
$\square \alpha_{9}$ & 3 & 3 & {$[1,11]$} \\
$\square \alpha_{10}$ & 100 & 50 & {$[25,75]$} \\
$\square \alpha_{11}$ & 50 & 20 & {$[10,60]$} \\
$\square \sigma$ & 0.3 & 0.3 & {$[0.1,0.6]$} \\
$\square P$ & 100 & 500 & {$[250,750]$} \\
\hline
\end{tabular}

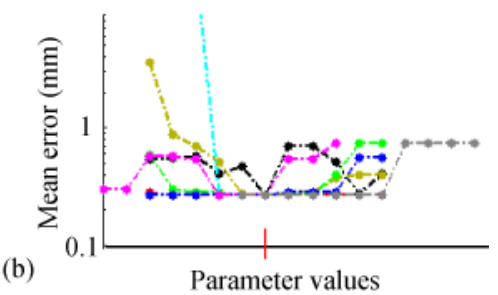

(b)

Parameter values

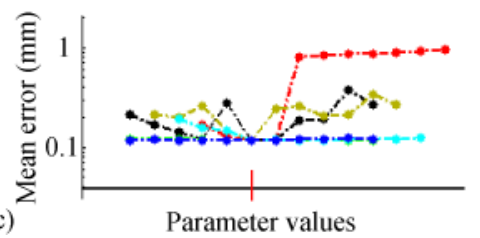

Fig. 2.

Shown in (a) is a table of the various parameters used in the methods for coarse localization (top group) and refinement (bottom group). (b) and (c) show errors when testing each parameter (color-codes in (a)) over the range specified in (a). The red hash mark indicates the final parameter value 


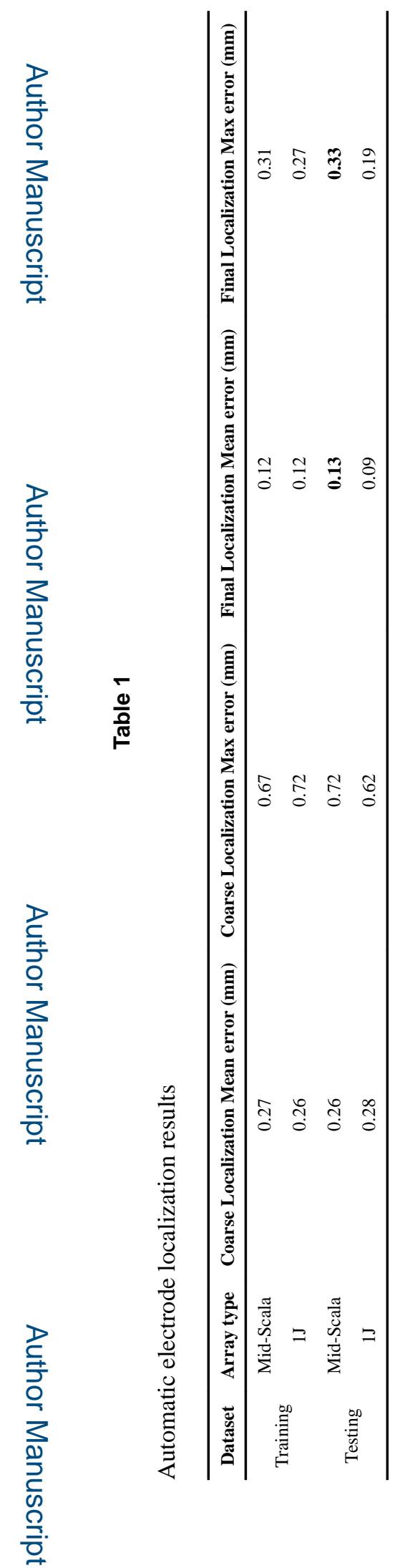

Med Image Comput Comput Assist Interv. Author manuscript; available in PMC 2016 October 01. 